Arq. Bras. Med. Vet. Zootec., v.68, n.5, p.1195-1200, 2016

\title{
Torção esplênica primária em um cão: relato de caso
}

\author{
[Primary splenic torsion in a dog: case report] \\ B.C. Ortiz, C.M. Oliveira, L.G. Teixeira*, M.C. Koch, V.S. Muller \\ Universidade Luterana do Brasil - Canoas, RS
}

\begin{abstract}
RESUMO
A torção esplênica isolada ou primária é uma enfermidade de ocorrência rara em cães, caracterizada pela torção do pedículo esplênico, sem que haja outra enfermidade concomitante. Os sinais clínicos e os exames laboratoriais são inespecíficos, porém a ultrassonografia, juntamente com o uso de Doppler colorido, tem sido importante para sugerir o diagnóstico, que é confirmado por meio de laparotomia exploratória. Este estudo relata o caso de um cão da raça Bulldog campeiro, cinco anos de idade, muito ativo, que recebia alimentação apenas uma vez ao dia. Este foi atendido no Hospital Veterinário da Universidade Luterana do Brasil (HV-Ulbra), com histórico de prostração, emagrecimento e anorexia intermitente havia 10 dias. Neste caso relatado, o exame ultrassonográfico com Doppler colorido foi imprescindível para o diagnóstico, que pôde ser confirmado posteriormente pela laparotomia exploratória. $\mathrm{Na}$ celiotomia, observou-se o baço aumentado e rotacionado, envolto com o omento, e ambos encontravam-se desvitalizados. Por essa razão, foram realizados os procedimentos de esplenectomia total e omentectomia parcial da porção acometida.
\end{abstract}

Palavras-chave: cão, baço, torção, ultrassonografia, laparotomia

\begin{abstract}
The individual or primary splenic torsion is a rare disease occurrence in dogs characterized by twisting the splenic pedicle, without any other concomitant disease. Clinical signs and laboratory tests are nonspecific, however, ultrasonography, along with the use of color Doppler, has been important to suggest the diagnosis is confirmed through exploratory laparotomy. This study reports the case of a 5year-old very active male Bulldog Campeiro, who received food once a day, which was attended at the Veterinary Hospital of Universidade Luterana do Brasil (HV ULBRA) with a history of prostration, weight loss and intermittent anorexia during 10 days. Ultrasonography with color Doppler exam was essential for the diagnosis, which could be confirmed later by exploratory laparotomy. In celiotomy it was observed the spleen increased in size and rotated, wrapped with omentum and both were found devitalized. For this reason, the procedures of total splenectomy and partial omentectomy of the affected portion were performed.
\end{abstract}

Keyword: dog, spleen,torsion, ultrasonography, laparotomy

\section{INTRODUÇ̃̃O}

A torção esplênica é uma afecção cirúrgica de ocorrência rara em cães, acomete principalmente cães de porte grande e raças de peito profundo, com alto nível de atividade (Goldsmid et al., 1994; Neath et al., 1997). A etiologia da torção esplênica isolada ou primária é desconhecida,

Recebido em 15 de outubro de 2015

Aceito em 29 de março de 2016

* Autor para correspondência (corresponding author)

E-mail: lucianateixeira@gmail.com mas acredita-se que esteja relacionada à torção/dilatação gástrica parcial ou de resolução espontânea, em que o estômago retorna à posição normal, enquanto o baço se mantém rotacionado ou tem seus ligamentos afrouxados, predispondo o órgão à torção (Stead et al., 1983; Mai, 2006).

As manifestações clínicas são inespecíficas, o que dificulta o diagnóstico, podendo ocorrer sinais de dor abdominal, aumento do pulso e da 
frequência respiratória, palidez das mucosas, vômito, anorexia, perda de peso, distenção abdominal, depressão e letargia, conforme relatam Goldsmid et al. (1994), Neath et al. (1997) e Reinhart et al. (2015). Nos achados laboratoriais, normalmente se pode observar anemia moderada, leucocitose, aumento da fosfatase alcalina e da ureia, hemoglobinemia e hemoglobinúria (Goldsmid et al., 1994), não caracterizando a patologia. A ultrassonografia abdominal, acompanhada de Doppler colorido, é valiosa no diagnóstico por permitir a identificação de esplenomegalia grave, além de alterações tanto no parênquima como no fluxo sanguíneo esplênicos (Saunders et al., 1998), direcionando para o tratamento cirúrgico.

O objetivo deste trabalho foi relatar um caso de torção esplênica primária em um cão da raça Bulldog campeiro, diagnosticado por meio de exame ultrassonográfico com Doppler colorido e confirmado mediante laparotomia exploratória. Não é do conhecimento dos autores a descrição de torção esplênica isolada na raça em questão.

\section{CASUÍSTICA}

Um cão da raça Bulldog campeiro, de cinco anos, sem histórico de doença prévia, pesando $21 \mathrm{~kg}$, foi encaminhado para atendimento no Hospital Veterinário da Universidade Luterana do Brasil (HV-Ulbra), apresentando anorexia intermitente, emagrecimento progressivo e leve prostração havia 10 dias. O proprietário relatou que o animal era muito ativo e que era alimentado uma vez ao dia, à noite. Na consulta, o paciente estava alerta, com tempo de preenchimento capilar de dois segundos, mucosas hipocoradas, temperatura retal de $39,2^{\circ} \mathrm{C}, 108$ batimentos cardíacos por minuto e 20 movimentos por minuto de frequência respiratória, algia abdominal e abdome rígido. À palpação abdominal era possível sentir massa firme em região lateral esquerda do abdome. $\mathrm{O}$ animal estava sendo tratado por outro veterinário com doxiciclina $(5 \mathrm{mg} / \mathrm{kg}$ bid) havia cinco dias, mas sem sinais significativos de melhora.

O hemograma completo revelou anemia macrocítica hipocrômica (hematócrito 23\%), sendo observados poiquilocitose e codócitos, leucocitose com neutrofilia sem desvio à esquerda $(35,700 / \mu \mathrm{L})$ e plaquetas dentro dos padrões de normalidade para a espécie
$(540,000 / \mu \mathrm{L})$. Na avaliação bioquímica sérica, os valores de creatinina, ureia e alanina aminotransferase (ALT) encontravam-se dentro dos valores basais, enquanto fosfatase alcalina (FA) se encontrava aumentada (217U/L). Para diagnóstico diferencial, solicitou-se exame parasitológico de sangue, que resultou negativo para hemoparasitas.

O paciente foi encaminhado para o setor de ultrassonografia do HV-Ulbra e submetido à varredura abdominal, após tricotomia e colocação de gel condutor, com transdutor convexo de baixa frequência $(5 \mathrm{MHz}) \mathrm{em}$ aparelho LOGIQ 500 PRO Séries. No exame, foi verificada esplenomegalia severa, estando o órgão dobrado sobre si mesmo, ocupando também o lado direito do abdome. O parênquima esplênico apresentava ecotextura difusa hipoecogênica e rendilhada (Fig. 1A). No exame modo B com Doppler colorido (Fig. 1B), nenhum fluxo sanguíneo foi detectado nas veias esplênicas. A gordura e o mesentério adjacentes apresentavam-se hiperecogênicos, e entre eles havia pequena quantidade de efusão peritoneal anecogênica (Fig. 1B). Os achados foram sugestivos de infarto e torção esplênica. $\mathrm{O}$ paciente foi encaminhado para laparotomia exploratória e esplenectomia total sem a distorção do órgão.

$\mathrm{Na}$ laparotomia exploratória, confirmou-se o quadro de torção esplênica. Foi realizada a exteriorização do baço, que se apresentava escurecido, desvitalizado, com morfologia severamente alterada, retorcido, envolto em porção do omento, também com aspecto desvitalizado (Fig. 3 e 4). O pedículo vascular esplênico, por sua vez, encontrava-se rotacionado em $360^{\circ}$. Na secção dos vasos do hilo esplênico, grande parte se encontrava avascularizada, sem sangramento. Optou-se pela remoção de $70 \%$ do omento pela ausência de pulsação e pelo seu aspecto desvitalizado. Devido ao grande espaço vazio intra-abdominal causado pela remoção do órgão, optou-se pela realização de gastropexia, a fim de se evitar torção vólvulo-gástrica secundária à cirurgia, conforme indicado pelos autores citados neste trabalho. O órgão foi encaminhado para exame histopatológico, que identificou congestão passiva aguda com lesão necro-hemorrágica, compatível com infarto crônico esplênico, e infiltrado neutrofílico focal junto à cápsula. 


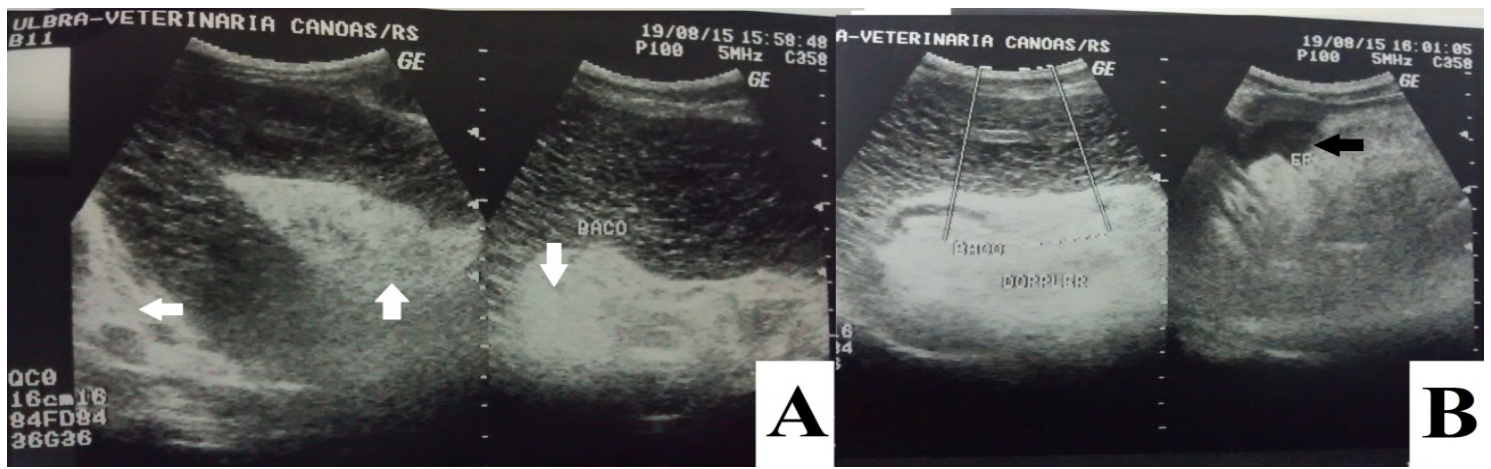

Figura 1. Curvatura esplênica com parênquima hipoecogênico rendilhado (A), ausência de fluxo sanguíneo ao Doppler colorido (B), gordura e mesentério hiperecogênicos/reativos (setas brancas), discreta efusão peritoneal adjacente (seta preta). HV-ULBRA, 2015 (Imagens 1-4 elaboradas pelos autores, cedidas pelo Hospital Veterinário ULBRA).

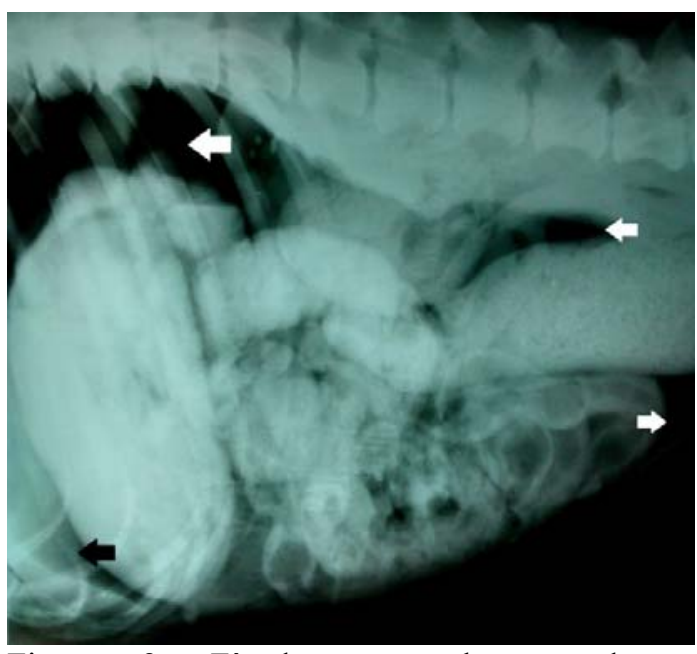

Figura 2. Fígado não ultrapassando o rebordo costal de T10 (seta preta), compatível com micro-hepatia, e presença de gás livre na cavidade (setas brancas) sugerindo pneumoperitôneo. Fonte: HV-ULBRA, 2015.

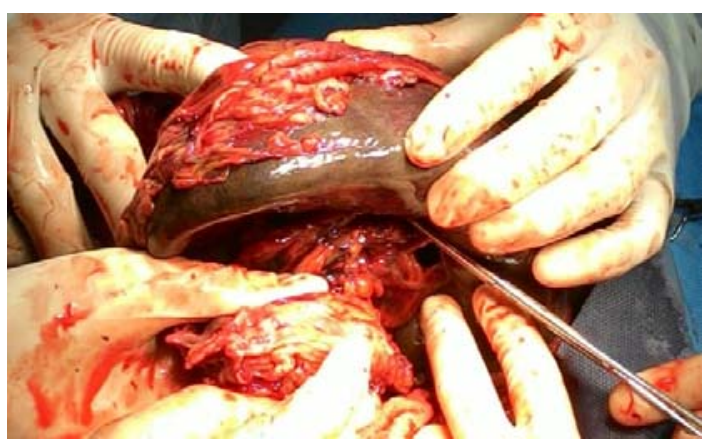

Figura 3. Baço com torçao de $360^{\circ}$ envolto por omento desvitalizado. Notar áreas amareladas no omento.

Fonte: HV-ULBRA, 2015.

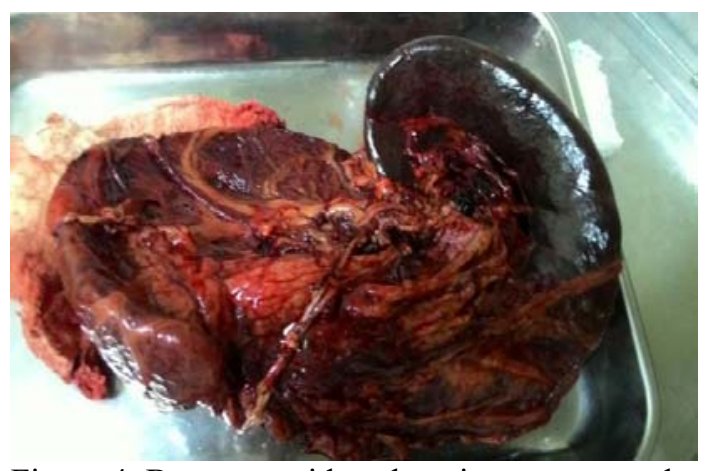

Figura 4. Baço retorcido sobre si mesmo, envolto por omento desvitalizado, após ser removido da cavidade abdominal.

Fonte: HV-ULBRA, 2015.

Ainda durante a cirurgia, foi iniciada transfusão de sangue total devido à perda de sangue no transcirúrgico, anemia prévia e queda progressiva da pressão arterial sistêmica e da saturação arterial de oxigênio. $O$ paciente permaneceu internado no HV- Ulbra por 10 dias para observação do pós-operatório e recuperação total do procedimento. Durante o período foram constantemente aferidos os parâmetros vitais do paciente, entre eles temperatura corporal, frequências cardíaca e respiratória, glicemia, lactato, tempo de preenchimento capilar, coloração de mucosas e pressão arterial sistólica com Doppler. Hemograma completo e bioquímica sérica foram avaliados diariamente durante o período de internação. No segundo dia após a cirurgia, houve um discreto aumento da creatinina de $1,2 \mathrm{mg} / \mathrm{dL}$ para $1,6 \mathrm{mg} / \mathrm{dL}$, que assim permaneceu até a alta, apesar da fluidoterapia constante. A ALT também apresentou um aumento de $67,6 \mathrm{Ul} / \mathrm{L}$ para 
124U1/L, retornando ao valor basal após dois dias.

$\mathrm{Na}$ prescrição, estavam incluídos cloridrato de tramadol $(5 \mathrm{mg} / \mathrm{kg}$ tid), ceftiofur $(2 \mathrm{mg} / \mathrm{kg}$ sid), metronidazol $(15 \mathrm{mg} / \mathrm{kg}$ bid), dipirona $(25 \mathrm{mg} / \mathrm{kg}$ tid), meloxicam $(0,2 \mathrm{mg} / \mathrm{kg}$ sid, depois $01 \mathrm{mg} / \mathrm{kg}$ sid), acetilcisteína $(140 \mathrm{mg} / \mathrm{kg}$, depois $70 \mathrm{mg} / \mathrm{kg}$ bid), suplementação vitamínica e mineral à base de ferro e fluidoterapia com solução de ringer com lactato de forma constante. Em ultrassonografia abdominal, realizada três dias após o procedimento cirúrgico, constatou-se presença de pneumoperitôneo, possivelmente decorrente da cirurgia, impossibilitando avaliação do parênquima hepático. Como alternativa, realizou-se radiografia abdominal, que confirmou pneumoperitôneo e evidenciou o fígado ocupando menos do que três espaços intercostais (Fig. 2), imagem compatível com micro-hepatia.

O paciente recebeu alta clínica com prescrição de protetores hepáticos (S-adenosilmetionina e silimarina) devido à alteração de ALT e microhepatia. Vinte dias após a cirurgia, todos os parâmetros haviam retornado aos valores de referência (hematócrito 38\%, leucócitos totais 14,100, ALT 38,2U/L e creatinina 1,2U/L).

\section{DISCUSSÃO}

A torção esplênica primária é afecção rara em cães, nunca tendo sido relatada em cão da raça Bulldog campeiro. Ocorre quando o baço gira em torno dos ligamentos gastroesplênicos, resultando em oclusão dos vasos esplênicos seguida por trombose vascular, congestão, hemorragia e necrose do baço (Saunders et al., 1998). Está mais frequentemente associada à dilatação vólvulo-gástrica do que a doenças esplênicas, porém, apesar de rara, pode ocorrer sem histórico de doença gástrica e esplênica prévia em 0,5\% dos cães acometidos (Mai, 2006). No estudo realizado por Saunders et al. (1998), todos os cães diagnosticados com torção esplênica eram jovens de meia-idade do sexo masculino e pertencentes a raças de grande porte, ou seja, características similares ao presente relato. Ainda, de acordo com este autor, a torção esplênica pode ocorrer tanto como um processo isolado quanto secundária à dilatação vólvulogástrica.
A etiologia de base é desconhecida, mas sugerese que esteja relacionada à distensão gástrica, à qual cães de raças grandes são mais predispostos, acompanhada ou não de vólvulo-gástrico, condição que é mais comum em animais de alto nível de atividade e que se alimentam apenas uma vez ao dia. Em estudo retrospectivo, Neath et al. (1997) identificaram que $21 \%$ dos cães acometidos com torção esplênica tiveram episódios de dilatação vólvulo-gástrica meses antes, corroborando esta teoria. Goldsmid et al. (1994) sugeriram que repetidos episódios de dilatação gástrica e consequente torção parcial deste órgão causam o estiramento e o afrouxamento dos ligamentos gastroesplênicos, ocasionando mobilidade e rotação esplênica. A rotação do pedículo esplênico, por sua vez, obstrui as veias esplênicas, provocando esplenomegalia. Essa era, muito provavelmente, a causa da esplenomegalia severa apresentada pelo paciente descrito, uma vez que nenhuma alteração no tecido gástrico foi visualizada na laparotomia exploratória. Ademais, tratava-se de um cão muito ativo, que era alimentado uma vez ao dia, com grande quantidade de ração, sem histórico de doença anterior, enquadrando-se nas descrições clássicas de apresentação desta doença.

A torção esplênica primária pode ocorrer de forma aguda ou crônica. A forma aguda é menos comum, sendo responsável por $30 \%$ dos casos (Ohta et al., 2009; Reinhart et al., 2015) e inclui dor abdominal severa, taquicardia, hipotensão e tempo de preenchimento capilar aumentado, como ocorre na síndrome dilatação vólvulogástrica. Na forma crônica, os sinais clínicos são inespecíficos e, muitas vezes, os animais são tratados para outras patologias, como anemia hemolítica imunomediada e erliquiose, antes de serem diagnosticados com torção esplênica (Millis et al., 1995; Ohta et al., 2009; Azevedo et al., 2011; Reinhart et al., 2015). Como descrito na literatura citada, o paciente relatado apresentava anorexia, letargia, dor à palpação abdominal, anemia, leucocitose e teve um diagnóstico prévio de hemoparasitose pelo primeiro veterinário que o atendeu. A doença progredia havia cerca de 10 dias, quando atendimento foi buscado no HV-Ulbra, o que condiz com dados da literatura em que cães demonstraram sinais de torção esplênica por até quatro meses antes do diagnóstico (Stead et al., 1983; Neath et al., 1997; Weber, 2000; Reinhart 
et al., 2015). O paciente apresentava anemia resultante do sequestro de hemácias pelo baço torcido e da inflamação em curso, uma vez descartadas outras possibilidades, como hemoparasitose e anemia hemolítica imunomediada, corroborando descrições da literatura (Schnier, 2010). A presença de poiquilocitose e codócitos estava relacionada à anemia grave com ativa regeneração, dado evidenciado pelo número de reticulócitos. $\mathrm{O}$ leucograma inflamatório, neste caso, poderia estar relacionado com a inflamação, e esta, por sua vez, poderia ser associada à baixa perfusão do parênquima esplênico e peritonite localizada secundária à torção esplênica, que foi demonstrada pelo mesentério reativo e pela efusão peritoneal, visualizados na ultrassonografia.

O baço do paciente apresentava-se severamente aumentado, retorcido sobre ele mesmo, estendendo-se para o lado direito. O parênquima apresentava-se hipoecoico, com ecotextura rendilhada. Adjacente a ele era possível visibilizar a gordura e o mesentério hiperecogênicos, além de pequena quantidade de efusão peritoneal, detectada no exame ultrassonográfico, achados compatíveis com os descritos por Penninck (2011) em casos de torção esplênica. Segundo Mai (2006), o mesentério no nível do hilo do baço é frequentemente marcado e hiperecoico. $\mathrm{O}$ órgão tem uma posição anormal e a efusão peritoneal pode estar presente, mas esplenomegalia com parênquima rendilhado não é específico de torção, pois esses achados podem ser observados na necrose, no infarto, entre outras causas. A quantidade de parênquima necrosado e de áreas de hemorragia depende da duração da torção, pois em alguns casos o parênquima pode estar normal.

Mai (2006) enfatiza que o uso do Doppler facilita o diagnóstico, demonstrando a falta de vascularização; porém, a ausência de fluxo sanguíneo também pode ocorrer devido à trombose da veia esplênica, mesmo quando ecos intraluminais não podem ser identificados na imagem devido à inerente falta de coágulos ecogênicos. A ultrassonografia com Doppler colorido possibilitou a detecção de ausência de fluxo sanguíneo no parênquima esplênico, enquanto imagens compatíveis com trombose não foram visibilizadas. Como diagnóstico diferencial, após esplenectomia, o órgão foi encaminhado para o setor de patologia do HVUlbra. No exame macroscópico e histopatológico, identificou-se apenas congestão passiva aguda com lesão necro-hemorrágica, compatível com infarto crônico esplênico, achados semelhantes com os citados por Saunders (1998) e Mai (2006).

\section{CONCLUSÃO}

A torção esplênica primária ou isolada é uma afecção pouco comum em cães e, normalmente, apresenta-se de forma crônica. $\mathrm{O}$ diagnóstico se baseia em sinais clínicos e exames complementares, como o ultrassom abdominal com avaliação do fluxo sanguíneo por meio do Doppler colorido, onde há esplenomegalia severa, estando o órgão dobrado sobre si mesmo, e ausência de fluxo sanguíneo. A laparotomia exploratória com visualização do baço rotacionado e de aspecto desvitalizado fundamenta o diagnóstico final. O tratamento é a esplenectomia total. Alto nível de atividade associado com baixa frequência diária de alimentação caracteriza-se como importante fator de risco para o desenvolvimento da torção esplênica primária. Cães de raças grandes com essas duas características estão, por sua vez, mais propensos a essa afecção.

\section{REFERÊNCIAS}

AZEVEDO, F.; VEIGA, C.C.P.; SCOTT, F.B. et al. Torção primária do baço em cães: relato de caso. Rev. Bras. Med. Vet., v.33, p.89-94, 2011.

GOLDSMID, S.E.; DAVID, P.E.; PECHMAN, R. Successful derotation of a splenic torsion in a racing greyhound. J. Small Anim. Pract., v.35, p.112-115, 1994.

MAI, W. The hilar perivenous hyperechoic triangle as a sign of acute splenic torsion in dogs. Vet. Radiol. Ultrasound, v.47, p.487-491, 2006.

MILLIS, D.L.; NEMZEK, J.; RIGGS, C.; WALSHAW, R. Gastric dilatarion-volvulus after splenic torsion in two dogs. J. Am. Vet. Med. Assoc., v.207, p.314-315, 1995.

NEATH, P.J.; BROCKMAN, D.J.; SAUNDERS, H.M. Retrospective analyse of 19 cases of isolated torsion of the splenic pedicle in dogs. $J$. Small Anim. Pract., v.38, p.387-392, 1997. 
OHTA, H.; TAKAGI, S.; MURAKAMI, M. et al. Primary splenic torsion in a Boston terrier. $J$. Vet. Med. Sci., v.71, p.1533-1535, 2009.

PENNINCK, D. Atlas of small animal ultrassonography. Rio de Janeiro: Guanabara Koogan, 2011. p.465.

REINHART, J.; SHERWOOD, J.; KUKANICH, K. et al. Chronic splenic torsion in two dogs. $J$. Am. Anim. Hosp. Assoc., v.51, p.185-190, 2015.

SAUNDERS, H.M.E.; NEATH, P.J., BROCKMAN, D. B-mode and Doppler ultrasound imaging of the spleen with canine splenic torsion: a retrospective evaluation. Vet. Radiol. Ultrasound, v.39, p.349-353, 1998.
SCHNIER, L.M. A case of splenic torsion with progressive anemia and thrombocytopenia. Can. Vet. J., v.51, p.527-529, 2010.

STEAD, A.C.; FRANKLAND, A.L.E.; BORTHWICK, R. Splenic torsion in dogs. J. Small Anim. Pract., v.24, p.549-554, 1983.

WEBER, N. Chronic primary splenic torsion with peritoneal adhesions in a dog: case report and literature review. J. Am. Anim. Hosp. Assoc., v.36, p.390-394, 2000. 